ISSN 1112-9867

http://www.jfas.info

\title{
PERFORMANCE ANALYSIS OF CAPACITY BASED COORDINATED MULTIPOINT (COMP) HANDOVER SCHEME IN LONG TERM EVALUATION-ADVANCED (LTE-A) NETWORK
}

\author{
A. L. Yusof ${ }^{1,2, *}$, M. A. Zainali ${ }^{1,2}$, N. Ya'acob ${ }^{1,2}$ and A. Idris ${ }^{1,2}$ \\ ${ }^{1}$ Faculty of Electrical Engineering, Universiti Teknologi MARA, 40450 Shah Alam, Selangor, \\ Malaysia \\ ${ }^{2}$ Wireless Communication Technology (WiCoT), Faculty of Electrical Engineering, Universiti \\ Teknologi MARA, 40450 Shah Alam, Selangor, Malaysia
}

Published online: 17 October 2017

\begin{abstract}
Coordinated Multipoint (CoMP) has been chosen in Release 11 as the best technique to improve both cell coverage and quality. However, the issue regarding the saturation on the system throughput in highly congested network leads to the system capacity overload. Thus, the existing CoMP handover scheme in LTE-A is not accommodating the conditions of the CoMP technique available. A capacity based CoMP handover scheme is proposed for better system capacity management. In CoMP, the coordination schemes are well developed in conjunction to improve overall quality for the user using MATLAB software. Performance analysis results show that capacity based CoMP handover scheme provides better Signal-to-Interference-plus-Noise Ratio (SINR) and reduces the number of unnecessary handover causes better handover performance.
\end{abstract}

Keywords: LTE-advanced; CoMP; handover scheme.

Author Correspondence, e-mail: laily012001@yahoo.com

doi: http://dx.doi.org/10.4314/jfas.v9i5s.25 


\section{INTRODUCTION}

\subsection{Background Study}

Since mobile users demands bigger network coverage, higher data rates, lower latencies and better throughput, LTE Release 10 seems to be an ideal improvement in order to meet those expectations. LTE is expected to have throughput of $1 \mathrm{~Gb} / \mathrm{s}$ in downlink (DL) and $500 \mathrm{Mb} / \mathrm{s}$ in uplink (UL). In order to maintain high performance by LTE-Advanced, multiple-input and multiple-output (MIMO) and orthogonal frequency-division multiplexing (OFDM) technology are introduced in the LTE downlink transmission while Single Carrier Frequency Division Multiple Access (SC-FDMA) is used in the uplink [1]. Those technologies focus more on multipath interference and improve spectral efficiency. Unfortunately, there is still no solution to mitigate the problem arising from the inter-cell interference especially experienced by the cell edge user. Thus, Coordinated Multipoint (CoMP) is introduced in Release 11 promised to provide better performance to the cell edge user due to severe Inter-carrier Interference (ICI) problems.

Furthermore, CoMP is famously known to increase cell coverage and cell throughput. According to [2], as soon as the location of user equipment (UE) is at cell edge neighbourhood, UE may experience multiple signals from from multiple cells sites that are geographically separated. But this scenario can be avoided in the way signals get coordinated. Hence, by applying several techniques in CoMP such as joint transmissions (JT) which convert the ICI into useful signal or a beam forming technique that avoids the ICI, the downlink performance might be outrageous. The resource scheduling based on joint maximum throughput is a promising technique that can lead to higher overall system throughput level but the complexity in the algorithm can affect the ability of UE to work well under certain condition and pressure. Besides resource scheduling technique, there were a lot of techniques were discussed among the researcher that used the concept of CoMP. Basically, techniques available in CoMP comprise of two main schemes, Joint Processing (JP) and Coordinate Beam forming/Coordinate Scheduling (CS/CB). Fig. 1 illustrates CoMP in distributed network architecture. JP is the most chosen technique among researcher due to its advantage of data is available at various point in CoMP set for exact time frequency resource for UE. Most of techniques use the same approaches such that scheduling information is 
needed when UEs data at the different Base Station (BS) that must be shared among them on backhaul network which is known as X2 interface [3,10-12].

It is well known that CoMP goals are to fully utilize of the whole system resources, to improve on network coverage and also to increase the quality for cell edge user. However, the implementation of CoMP in the uplink facing several challenges such as appropriate cluster cooperating BS have to be identified, the limitation on the maximum distance of cooperating BS and issues from the backhaul if centralized decoding is applied. Meanwhile in downlink CoMP, the complexity can be managed by forming smaller cooperation cluster in a large-scale network but there are solution needed in order to reduce feedback delay, integration of CoMP in higher layers and flexibility on the formation of cooperation cluster [5].

Handover can be defined as a mechanism that's maintains an ongoing call or data session while the user is moving from one cell to another. When a UE is moving from a cell to one of its neighboring cell, the UE switches its connections from serving BS to the target BS in a way to avoid call termination [6-7]. A handover algorithm or scheme is needed for making a handover decision. A handover will be triggered if the statements specified by handover algorithm are fulfilled. For LTE-A, hard handover is synonym to the system. The concept of break before make connections is applied in hard handover. In other meaning, UE must terminate its connection with source BS before establishing a new connection with the target BS. The reason of LTE network supports hard handover is to reduce delay due to the signaling overhead among BSs and the complexity of the LTE network architecture. Meanwhile in soft handover, the connection of the source BS is terminated after the connection to the target BS is engaged. Soft handover is widely used in $3 \mathrm{G}$ network and within. Although its advantages are high reliability due to the decrease the possibility of call drop caused by ping pong handover, more resource is needed in downlink especially for the code resource and increases the amount of signaling that is required between BSs [7]. 


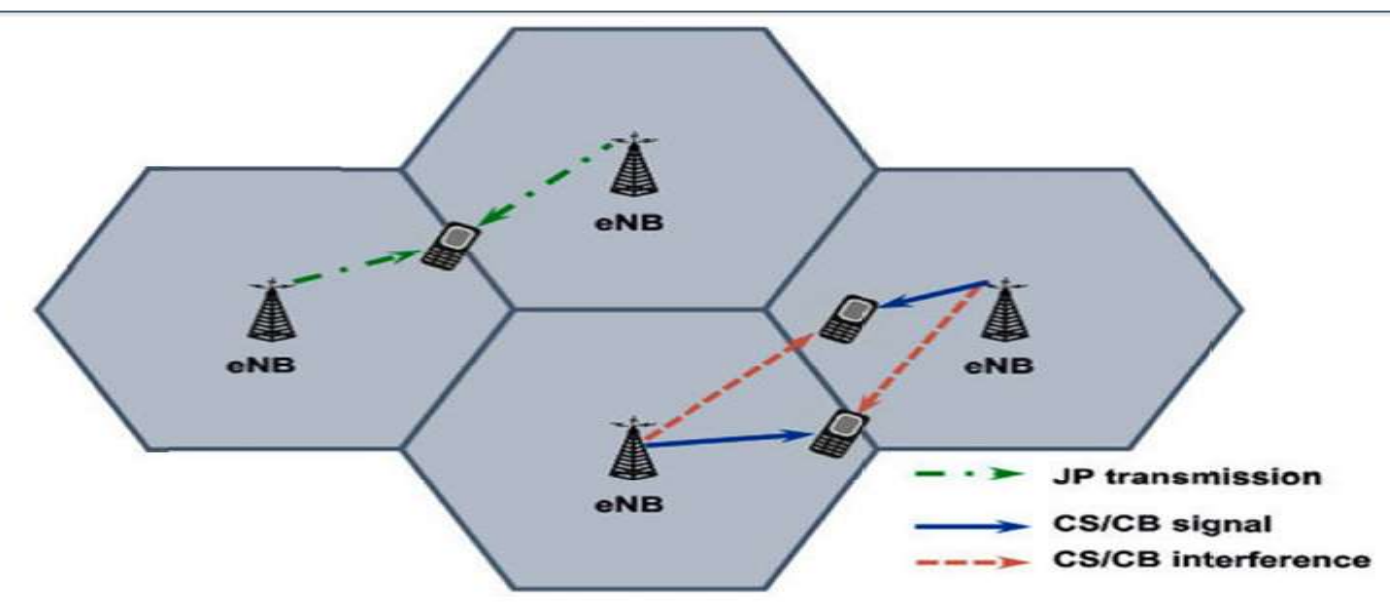

Fig.1.Techniques of CoMP in distributed network

In this paper, a handover scheme known as capacity based CoMP handover schemes is proposed, analyzed and compared with existing handover schemes. The rest of the paper can be categorized as follow. Section 1 explains detailed description of the capacity based CoMP handover schemes in LTE-A network followed by results and discussion described in section 2.Section 3 draws the conclusion.

\subsection{Capacity Based CoMP Handover Scheme}

In capacity based handover scheme, 3 important parameters are involved which are handover margin $(\mathrm{HOM})$, measurement period and RB utilization value. HOM is fixed variable that correspond to the threshold for the difference in SINR between serving cell and target cell. Measurement set is a time period that is used for checking the handover condition periodically. To describe the cell's capacity, RB utilization value is examined. If the value of RB utilization is high, it indicates the cell become saturated, where cell reselection needs to be considered when number of UE to be handed over increase. Meanwhile, when the cell is having a lower RB utilization value, the cell is able for accommodating more incoming UEs [2]. Besides that, double filtering of CCS and CTP selections is done to focus on the quality of target cells. The reason of regulating the RB utilization value in CCS selection is to reduce unnecessary feedbacks from UE to serving cell and to make sure the radio resources are efficiently used in the system.

Hence, based on CoMP system model of 3GPP LTE-Advanced [13], there are four elements had to be considered in order to make a decision in CoMP handover scheme. Those elements are serving cell, measurement set, CoMP coordinated set (CCS) and CoMP transmission 
points (CTP) [9]. Serving cell has tasks to maintain the connection of each UE to the network and deeply involved in making the handover decision. A group of cells named measurement set whose SINR and RB utilization value can be received by UE to the serving cell for choosing the selection of CCS. Meanwhile, CCS is known as a set of cells that are selected by serving cell form the measurement set. Lastly, CTP is a set of cells from CCS that is chosen by serving cell and used to send data directly to UE in downlink transmission [8]. Fig. 2 shows the illustration of the CoMP system model[4].

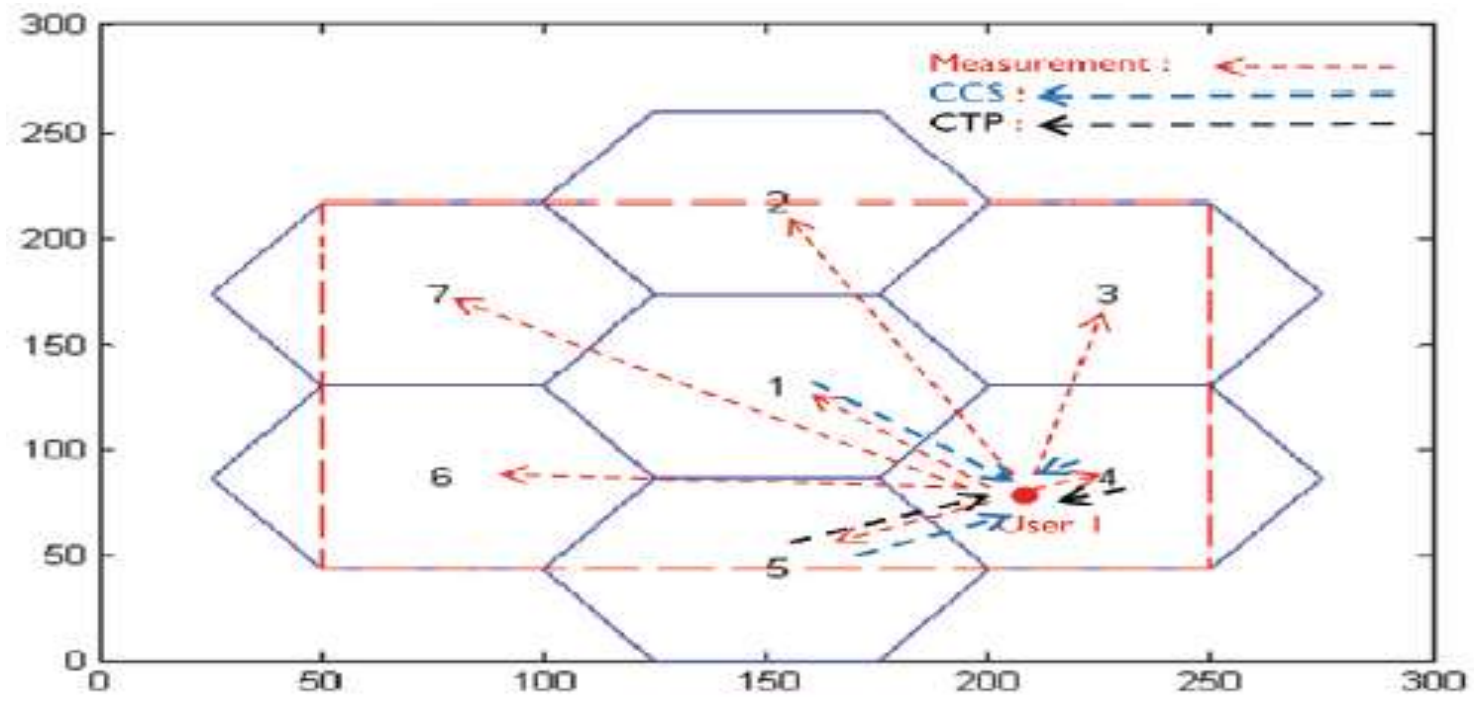

Fig.2.CoMPsystem model in LTE-A network

\section{RESULTS AND DISCUSSION}

The numerical and simulation results of capacity based CoMP handover scheme are presented and compared with existing handover scheme.

\subsection{Numerical Results}

In order to determine the size of CCS and CTP, the relationship between SINR and distance of UE from BS is studied as seen in Fig.3. Variation of distance with different step size will determine the size of cluster that will correspond to CCS and CTP selection. 


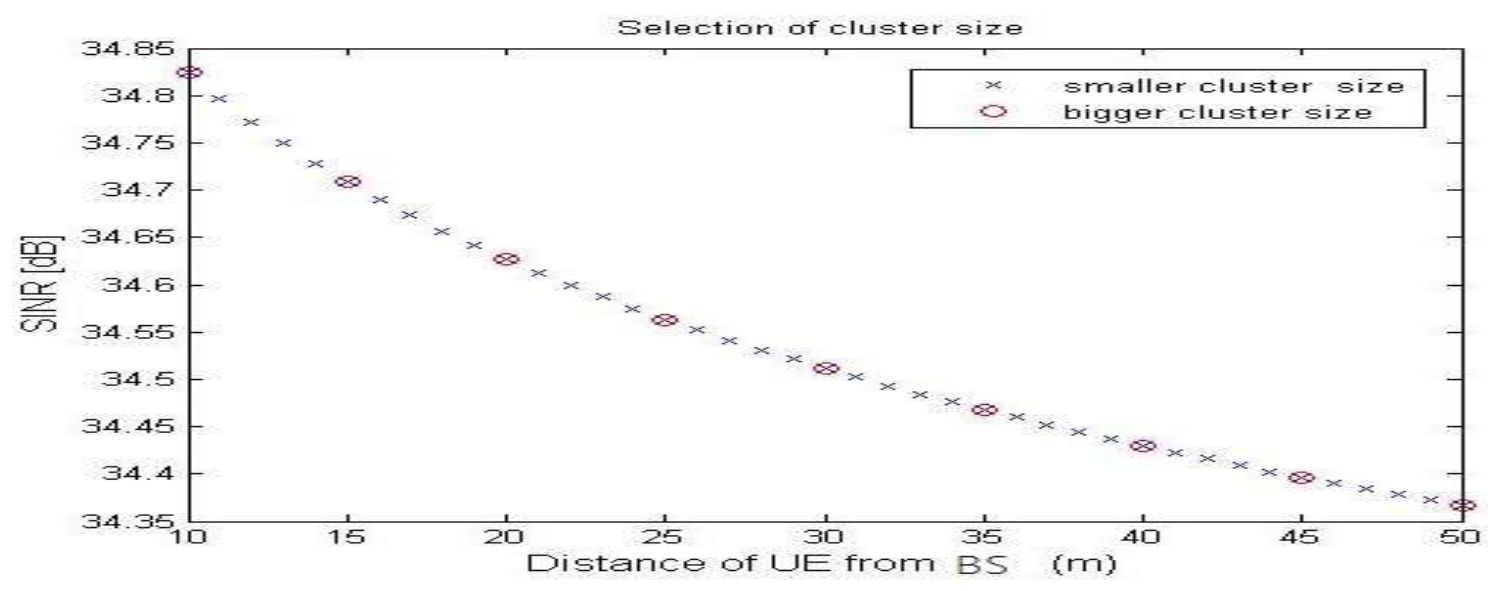

Fig.3. Selection of CCS and CTP

From Fig.3, the blue x marks indicate the smaller cluster size which represents CTP in this scheme as they have smaller step size while the red circle marks that indicate bigger cluster size. As the range of SINR between two consecutive red circles is bigger than that of the two consecutive blue $\mathrm{x}$ marks, the size affects cluster becomes bigger. Due to high signal link cost experienced by UE between two consecutive distances, the margin of SINR is not significantly varied in the selection of cluster size. When CoMP is deployed, CCS and CTP will be formed. As the size of CCS to CTP becomes smaller in cluster size, the signal link will be reduced. Meanwhile with the existing handover scheme, UE will calculate all the distance from all BS without considering the signaling cost. In consequences, the SINR in CTP is better. Due to the chosen lowest RB utilization value in CCS, the capacity of user with hotspot threshold value $(H d)$ will increase and causes the number of handover to decrease in the simulation result. 


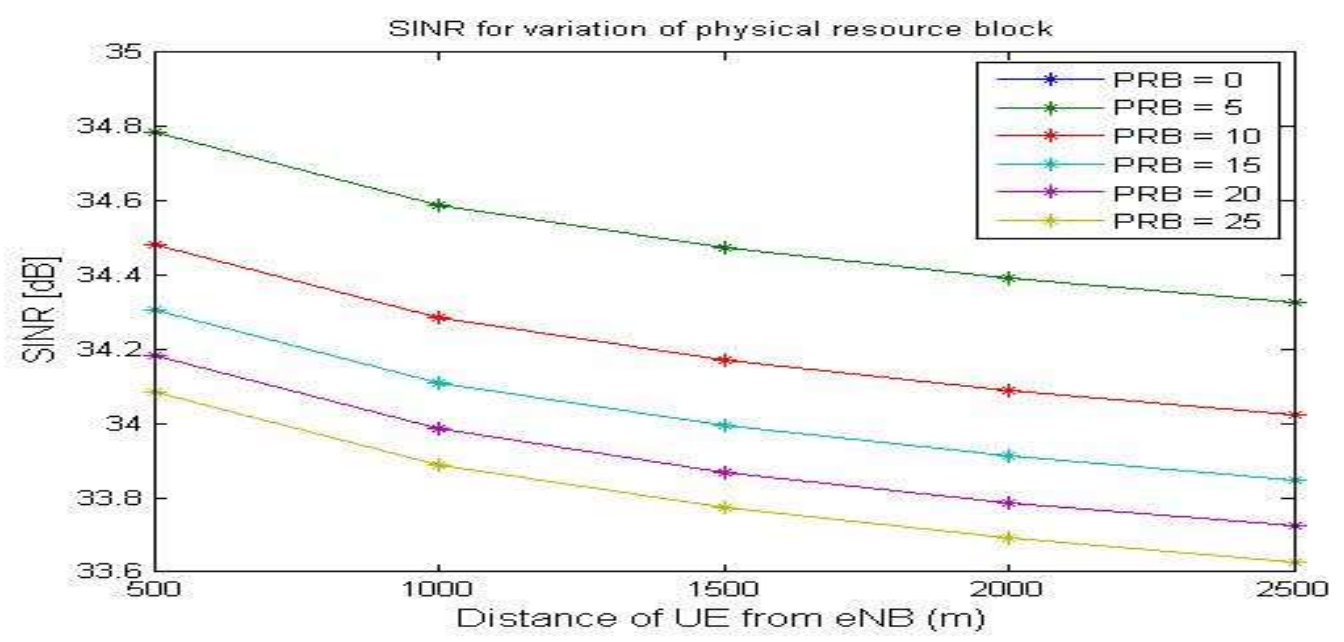

Fig.4. SINR versus distance of UE from BS with variation of PRB

Fig.4 shows correlation between SINR versus distance of UE from BS varies from 500m to $2500 \mathrm{~m}$ with variation of PRB. The maximum number of PRB is from 0 to 25 . At a distance of UE from BS equivalent to $2500 \mathrm{~m}$, the value of SINR is at the lowest with the number of available PRB reaching maximum. Resource block is the minimum unit of data transmitted. When the maximum PRB is occupied by UE, there will be a heavy traffic data being transmitted in downlink LTE-A network causes inter-cell interference increasing. In addition, there will be a downfall on the throughput per UE as the PRBs are shared among all the UE nearby.

\subsection{Simulation Results}

Fig. 5 shows the total number of handover of capacity based CoMP scheme and the existing handover scheme. From the graph, it can be said that when the number of user increases, the number of handover also increases. But, when capacity based CoMP scheme is applied denoted as yellow bar, the number of handover is significantly reduce than the number of handover experienced by UE without capacity based CoMP denoted as pink bar.

Meanwhile in Fig.6 shows total number of handover of capacity based CoMP handover scheme and the existing handover algorithm along the variation of simulation time. The graph shows the pattern of capacity based CoMP scheme is inconsistent. As at the time interval from $100 \mathrm{~s}$ to $500 \mathrm{~s}$, the total number of handover experienced by UE is increasing dynamically but when the time reaches 600 s till $1000 \mathrm{~s}$, the total number of handover is decreasing. Compared to the existing handover scheme, the pattern shows the total number of handover is constantly 
increasing with the simulation time. This is because capacity based CoMP scheme limits the chance that UEs camp on the target cell with the best radio signal quality when making a handover decision. Compared to the existing handover scheme, UE is getting more chances to encounter more handover condition as the capacity on the target channel is not limited. As a result, it leads to more number of handover in the result. If the number of available resources is bigger than the hotspot threshold $(H d)$, the current serving cell will formed CCS into the target cell based on lowest RB utilization value. In target cell, CoMP technique is applied where CCS will check the current channel available. If the hotspot channel is fully occupied, it will not initiate the handover process. In contrast, with the existing handover scheme, target cell is not considering the channel availability. As soon as target cell is found, it will initiate handover process and causes the number of drop call to increase.

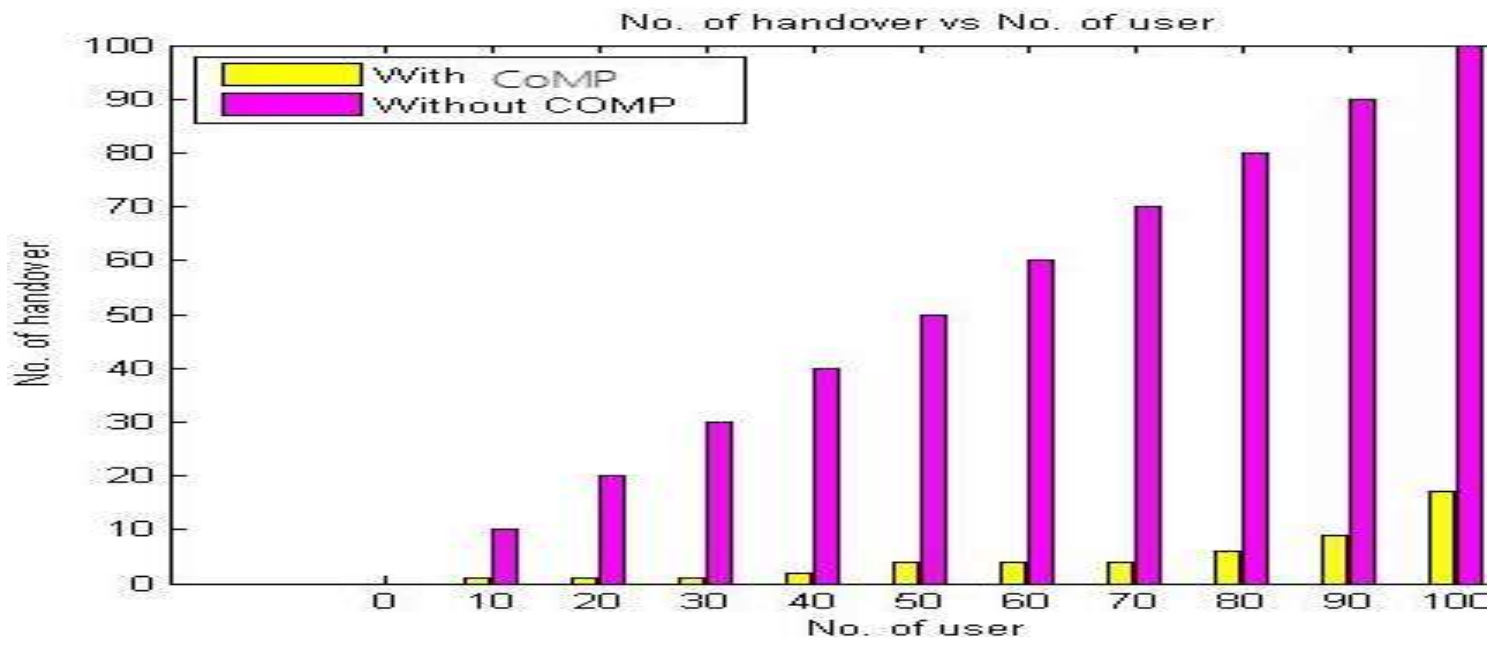

Fig.5. Graph of number of handover versus number of user 


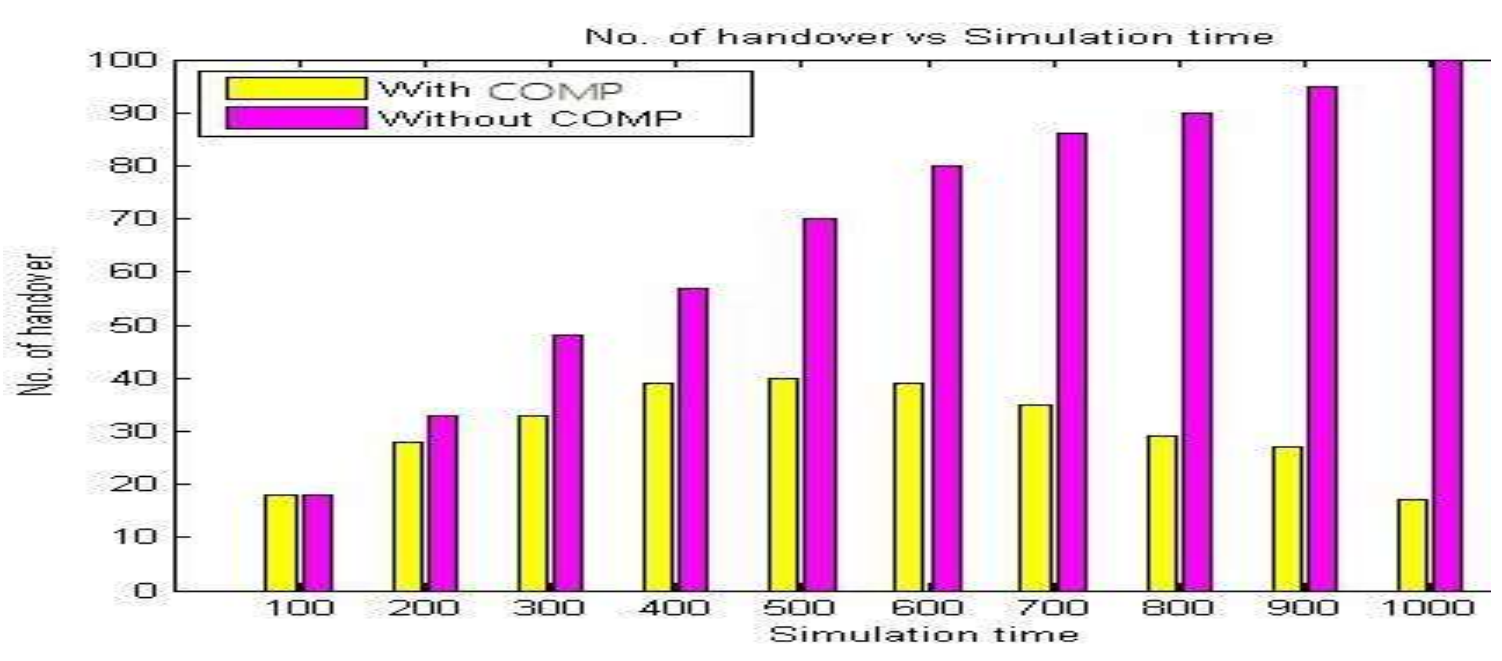

Fig.6. Graph of number of handover versus simulation time

In this scheme, the simulation system model is done with the aid of MATLAB software in order to analyze the performance of capacity based CoMP handover schemes in LTE-A network. The research concentrates on simulation process based on the proposed scheme under certain assumptions. The implementation in the real network is not being considered as femtocell LTE is not being deployed yet in Malaysia. The simulation is based on 33 clusters with 7 cells per cluster. The total number of BS is 231 and each BS is located at the center of each hexagonal cell structure. During simulation, UE is randomly position on the system model. In addition, the speed of UE is kept constant throughout the simulation which is 120 $\mathrm{km} / \mathrm{h}$ in the direction towards the south east of the system model. Fig.7 shows the designed cellular network. The blue dot indicates the centre base station of 7 cells per cluster and red ' $x$ ' marks indicates 100 user equipments. Simulation time was set up for 1000 s. The channel will be released by the UE after call finished and will terminate at the end of the simulation time. 


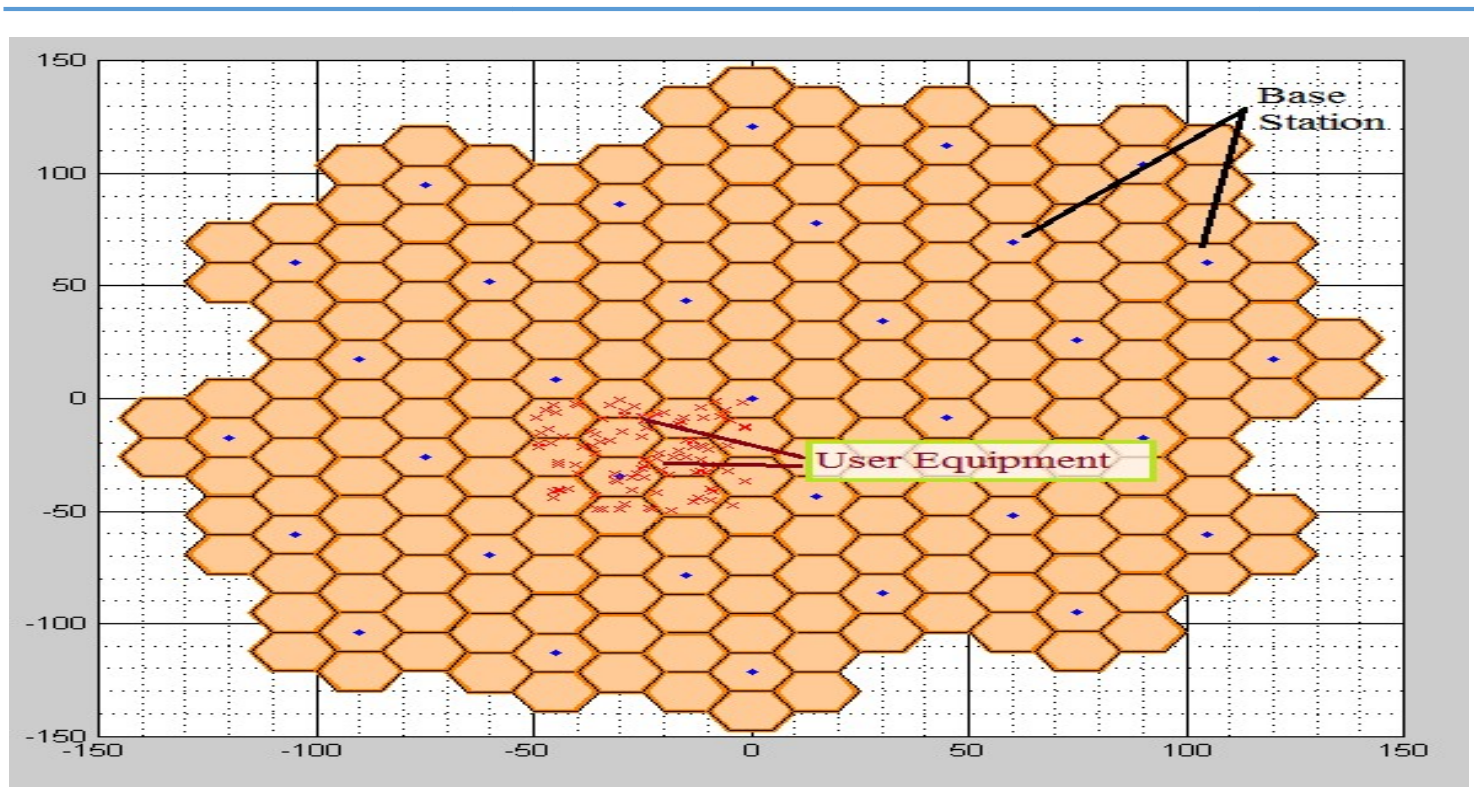

Fig.7. Simulation system model

\subsubsection{Signal to Interference to Noise Ratio}

As the transmission of data between UE and BS is based on SINR, UE gets an opportunity to select the serving BS based on received SINR and minimum path loss. The radio channel is modeled with Cost 231 Hata model for path loss. SINR, path loss and distance of UE from BS can be calculated by following equations:

Mobile antenna correction factor $=(1.1 * \log 10(f)-0.7) * h m-(1.56 *$

$\log 10(h b)-0.8)$

wherefis the carrier frequency, hmrepresents the height of UE (in meter) and $h b$ is the height of BS (in meter).

From the value of mobile antenna correction factor, the path loss between UE and BS can be obtained as in Equation (2):

Path loss $=$

$46.3+33.9 * \log 10(\mathrm{f})-13.2 * \log 10(\mathrm{hb})-$ Mobile antenna correction factor + $(44.9-6.55 * \log 10(\mathrm{hb})) * \log 10($ min_posBSc_posdist_min $(\mathrm{j}))$

wheremin_pos $B S c \_$posdist_min (j) is the minimum distance of UE from BS (in meter).

The equation of SINR is determined based on corresponding value of path loss as stated in Equation (3). 
$\operatorname{SINR}(\mathrm{j}, \mathrm{t})=\left(\operatorname{Ptotal} * 10^{\wedge}(\operatorname{Pathloss}(\mathrm{j}, \mathrm{t}) / 10)\right) /(\operatorname{PRB}(\mathrm{ICI}+\mathrm{No}))$

wherePtotalis total BS transmit power (in $\mathrm{dB}$ ), $P R B$ represents the number of available physical resource block, No is the thermal noise (in Watts) and ICI is the inter-cell interference (in Watts). Table 1 shows the summarization of the simulation parameters used in the following expressions.

Table 1. Simulation parameters [8]

\begin{tabular}{cc}
\hline Parameter & Assumption/Value \\
\hline Radius of BS & $1 \mathrm{~km}$ \\
Power transmit, Ptotal & $43.01 \mathrm{dBm}$ \\
Maximum number of PRBs & 25 \\
Frequency, $f$ & $2 \mathrm{GHz}$ \\
Height of eNB, $h b$ & $50 \mathrm{~m}$ \\
Height of user terminal, $h m$ & $5 \mathrm{~m}$ \\
Thermal noise, $N o$ & $7.24436 \mathrm{uW}$ \\
Inter-cell Interference, $I C I$ & $0.005 \mathrm{~W}$ \\
\hline
\end{tabular}

\subsubsection{Relationship between distance and SINR}

Distance of UE from BS is essential to calculate SINR, especially when UE is randomly positioned in the cell architecture. From the simulation system model, BS position is fixed with respective $\mathrm{x}$ and $\mathrm{y}$ coordinate and denoted as $\mathrm{BSc}$ pos $(\mathrm{j}, \mathrm{s})$ and UE in this case is UE_pos $(\mathrm{j}, \mathrm{s})$ where actual distance can be calculate inEquation (4).

$U E_{-}$posBS_posdist $(j, s)=\left(\left(U E_{-} \_ \text {pos }(\mathrm{j}, 1)-\mathrm{BSc} \_\operatorname{pos}(\mathrm{s}, 1)\right)^{\wedge} 2+\left(\mathrm{UE} \_\right.\right.$pos $(\mathrm{j}, 2)-$ BSc_pos $\left.(\mathrm{s}, 2))^{\wedge} 2\right)^{\wedge}(0.5)$

where $U E \_$pos is the position of UE in the cell architecture and $B S c \_p o s$ is the position of BS in the cell architecture. In CoMP, UE is always camp on the nearest BS by using equation in 5 .

$U E \_p o s B S \_p o s d i s t \_\min (j) \operatorname{ServBS}(j, t)=\min \left(\mathrm{MS} \_p o s B S c \_p o s d i s t_{(j, 1: 231))}\right.$

where $M S \_$pos $B S c \_$posdistis the distance between UE and BS (in meter).

After the distance is calculated, the path loss is measured using Equation (1) and (2)with the respect of given simulation time and yields SINR in Equation (3)for every UE is obtained. 


\subsubsection{Flowchart of Capacity Based CoMP Handover}

The handover scheme begins with the cell selection when UE enters the network by camping on the nearest BS on the cell architecture based on the SINR in the measurement set that is instructed by the previous serving cell. In order to select CCS, the serving cell chooses a set of cells with the lowest RB utilization value. Next, the selection of CTP will be done by the serving cell based on a set of cells with the highest SINR from CCS until it satisfied in Equation (6). SINR > Threshold

6)

A handover is triggered when the condition based on Equation (7) is satisfied.

SINRctp $>$ SINRsc $+\mathrm{HOM}$

whereSINR ctp and SINRsc are the SINR received by a UE from target cell in the CTP and the serving cell respectively.

When UE experienced handover to other BS, the serving cell will activates all cells in CTP to cancel all current CTP transmissions. Then, serving cell will instruct UE to handover to target cell. Hence, UE will detach the network and camps on new serving cell within simulation time. Fig. 8 shows the detail description of the process of capacity based CoMP handover scheme. 


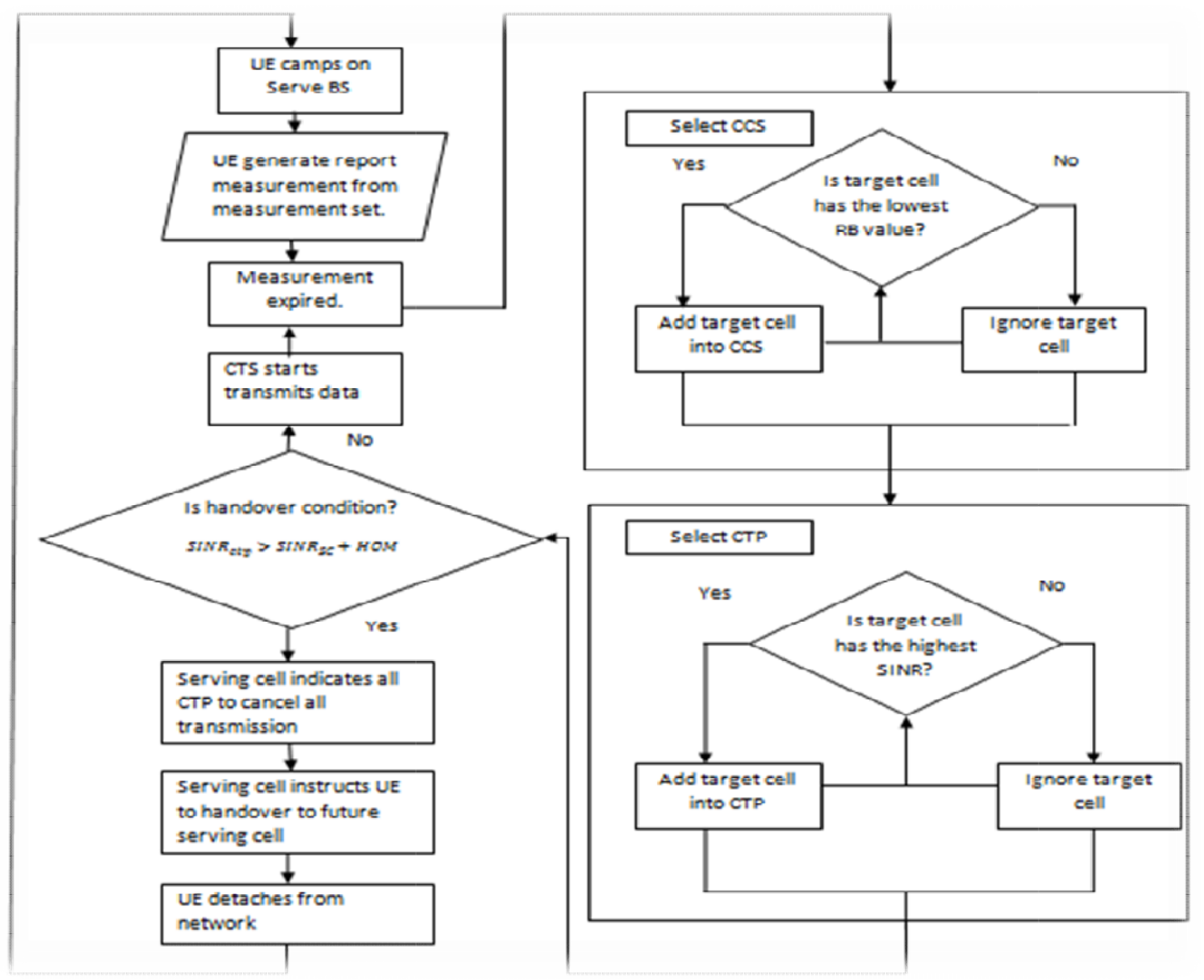

Fig.8. Flowchart of capacity based handover scheme

\section{CONCLUSION}

Capacity based CoMP handover scheme is proposed and analyzed in this paper. The performance is analyzed in both simulation and numerical results. The simulation results shows that with limitation of traffic hotspot threshold $(H d)$, number of handover is reduced compared to the existing handover scheme. Besides that, from the numerical result, the signaling cost is reduced due to the selection of CCS and CTP.Hence, the proposed scheme provides better SINR and led to the decrease of inter-cell interference experienced in the cell edge user. In addition, the number of unnecessary handover is reduced causes better handover performance. However, this handover scheme can be improved in order to reduce the overhead of information exchange and signaling in CoMP scenario. Future studies includes an improved capacity integrated CoMP handover scheme can be considered and more parameters to evaluate the performance of this handover scheme such as the determination on cell 
throughput and system delay.

\section{ACKNOWLEDGEMENTS}

This paper is part of research work that supported by Faculty of Electrical Engineering, UiTM Shah Alam.

\section{REFERENCES}

[1] Holma H, Toskala A. LTE for UMTS-evolution to LTE-advanced. New Jersey: John Wiley and Sons, 2011

[2] Heba R A, Essam S, Hassan M. Elkamchouchi. Comp-JT with dynnamic cell selection, global precoding matric and IRC receiver For LTE-A. International Journal of Mobile Networks, 2013,7(3):27-45

[3] Lin C C, Sandrasegaran K, Reeves S. Handover algorithm with joint processing in LTE-advanced. In 9th International Conference on Electrical Engineering/Electronics, Computer, Telecommunications and Information Technology, 2012, pp. 1-4

[4] Lin C C, Sandrasegaran K, Zhu X, Xu Z.Limited comp handover algorithm For LTE-advanced.Journal of Engineering,2013, 2013:1-9

[5] Ali M S. On the evolution of coordinated multipoint (CoMP) transmission in LTE-advanced. International Jourrnal of Future Generation Communication and Networking, 2014, 7(4):91-102

[6] Irmer R, Droste H, Marsch P, Grieger M, Fettweis G, Brueck S, Mayer HP, Thiele L, Jungnickel V. Coordinated multipoint:Concepts, performance, and field trial results. IEEE Communication Magazine, 2011, 49(2):102-111

[7] Hussein Y S, Ali B M, Varahram P, Sali A.Enhanced handover mechanism in long term evolution (LTE) networks. Scientific Research and Essays, 2011, 6(24):5138-5152

[8] Nakano A, Saba T. A handover scheme based on signal power of coordinated base stations for CoMP joint processing systems. In 8th IEEE International Conference onSignal Processing and Communication Systems, 2014, pp. 1-6

[9] Xiaodong X, Xin C, Jingya L. Handover mechanism in coordinated multi-point transmission/reception system. ZTE Communications, 2010, 1 
[10]Al Kim H. Extending the LTE-Sim for LTE advance with CoMP and relaying in heterogeneous 4G mobile networks. Sydney: University of Technology, 2014

[11]Terng L J, Hashim F. Performance analysis of coordinated multipoint (CoMP) in long term evolution advanced.In 2nd IEEE International Symposium on Telecommunication Technologies,2014, pp. 379-383

[12] Apilo O, Lasanen M, Mämmelä A.Energy-efficient dynamic point selection and scheduling method for intra-cell CoMP in LTE-A. Wireless Personal Communications, 2016, $86(2): 705-726$

[13] Huda A M R, Zairi I R. Novel scheduling algorithm for optimizing real-time multimedia performance in long term evolution-advanced. Turkish Journal of Electrical Engineering and Computer Sciences, 2017, 25(1):247-253

\section{How to cite this article:}

Yusof A L, Zainali M A, Ya'acob N, Idris A. Performance analysis of capacity based coordinated multipoint (comp) handover scheme in long term evaluation-advanced (lte-a) network. J. Fundam. Appl. Sci., 2017, 9(5S), 352-366. 